\title{
Genome Sequence Data of Six Isolates of Phytophthora capsici from Mexico
}

\author{
Alfredo Reyes-Tena, ${ }^{1,2}$ José C. Huguet-Tapia, ${ }^{2}$ Kurt H. Lamour, ${ }^{3}$ Erica M. Goss, ${ }^{2,4}$ \\ Gerardo Rodríguez-Alvarado, ${ }^{1}$ Gerardo Vázquez-Marrufo, ${ }^{5}$ Ricardo Santillán-Mendoza, ${ }^{1}$ \\ and Sylvia P. Fernández-Pavía ${ }^{1 \dagger}$ \\ ${ }^{1}$ Laboratorio de Patología Vegetal, Instituto de Investigaciones Agropecuarias y Forestales, Universidad \\ Michoacana de San Nicolás de Hidalgo, Tarímbaro, Michoacán, 58880, México \\ ${ }^{2}$ Department of Plant Pathology, University of Florida, Gainesville, FL, 32611, U.S.A. \\ ${ }^{3}$ Department of Entomology and Plant Pathology, University of Tennessee, Knoxville, TN, 37996, U.S.A. \\ ${ }^{4}$ Emerging Pathogens Institute, University of Florida, Gainesville, FL, 32610, U.S.A. \\ ${ }^{5}$ Centro Multidisciplinario de Estudios en Biotecnología, Facultad de Medicina Veterinaria y Zootecnia, \\ Universidad Michoacana de San Nicolás de Hidalgo, Morelia, Michoacán, 58880, México
}

\section{Funding}

A. Reyes-Tena thanks the Consejo Nacional de Ciencia y Tecnología of Mexico (CONACYT) for providing a doctoral scholarship (number 429123) and the mobility scholarship.

\section{Keywords}

genomics, oomycete effectors, oomycete-plant interactions

\section{Genome Announcement}

Phytophthora capsici is an important oomycete plant pathogen on vegetable crops. It is the causal agent of the principal disease of Capsicum annuum worldwide, and can infect many other economically important vegetables in the Solanaceae and Cucurbitaceae families (Reis et al. 2018). Disease can be on all portions of the host plants, including root rot, stem necrosis, foliar blight, and fruit rot (Candole et al. 2012). The main virulence factors of this pathogen are secreted RXLR and Crinkler (CRN) effector proteins that counteract the plant defense response and facilitate infection (Kong et al. 2017; Stam et al. 2013). $P$. capsici has shown great adaptability to fungicides and new hosts. Analysis of the reference genome suggested that the loss of heterozygosity could be involved in fixing alleles and facilitating adaptation to its environment (Lamour et al. 2012). To expand upon the available sequenced genomes, we assembled six genomes of isolates of $P$. capsici from four hosts in Michoacan, Mexico. These isolates are from hosts that are not represented in previous studies of $P$. capsici (Hu et al. 2018).

$P$. capsici isolates were obtained from Cucurbita pepo (CPV-262 and CPV-267, from the same field), Capsicum annuum (CPV-270 and CPV-277), C. chinense (CPV-302), and C. pubescens (CPV-219) in Michoacan, Mexico. The isolates were cultivated in V8 agar. Genomic DNA was extracted following the method described by Saghai-Maroof et al. (1984). For sequencing, $2 \times 150$-bp paired-end PCR-free Illumina libraries with an average insert size of $250 \mathrm{bp}$ were constructed using the KAPA hyperprep kit according to the manufacturer's directions. Sequencing was performed on a HiSeq $X$ device at Admera Health according to current Illumina protocols. The sequences obtained had the following

\section{†Corresponding author: S. P. Fernández-Pavía; fernandezpavia@ hotmail.com}

The author(s) declare no conflict of interest.

Accepted for publication 10 June 2019.

(c) 2019 The American Phytopathological Society 
Table 1. General metrics of the genome assemblies of Phytophthora capsici

\begin{tabular}{lcccccccc} 
Isolate & $\begin{array}{c}\text { Total length } \\
\text { (Mb) }\end{array}$ & $\begin{array}{c}\text { Number } \\
\text { of contigs }\end{array}$ & N50 & L50 & $\begin{array}{c}\text { Total predicted } \\
\text { proteins }\end{array}$ & $\begin{array}{c}\text { RXLR } \\
\text { content }\end{array}$ & $\begin{array}{c}\text { CRN } \\
\text { content }\end{array}$ & NPP1 \\
CPV-219 & 58.88 & 7,580 & 14,052 & 1,184 & 25,102 & 379 & 230 & 83 \\
CPV-262 & 40.77 & 11,754 & 4,558 & 2,492 & 19,275 & 263 & 121 \\
CPV-267 & 52.19 & 11,832 & 6,599 & 2,287 & 23,579 & 341 & 183 \\
CPV-270 & 58.29 & 8,363 & 11,645 & 1,422 & 25,004 & 371 & 213 & 79 \\
CPV-277 & 53.43 & 9,341 & 9,423 & 1,599 & 23,404 & 350 & 181 \\
CPV-302 & 56.90 & 9,277 & 9,970 & 1,652 & 24,322 & 353 & 187 \\
\hline
\end{tabular}

read depth per genome: CPV-219 = 111 $\times$, CPV-262 = 62x, CPV-267 = 74x, CPV-270 = 80x, CPV-277 $=77 \times$, and CPV-302 $=98 \times$. The raw sequences were quality filtered and adapter trimmed with Trim-Galore (Babraham Bioinformatics). Platanus-allee version 2.0.1 was used for the de novo genome assembly (Kajitani et al. 2014). To remove alternative heterozygous contigs and to obtain less fragmented assemblies, the Redundans pipeline was applied to each genome (Pryszcz and Gabaldón 2016). Contigs shorter than $1 \mathrm{~kb}$ were filtered out of the final assembly. General statistics of the genome assemblies were assessed with QUAST (Gurevich et al. 2013). The GC content ranged between 49.85 and $49.96 \%$ among genomes. The genome sizes, number of contigs assembled, and N50 and L50 values are shown in Table 1.

GeneMark-ES was used for the $a b$ initio prediction of genes and to obtain protein sequences (Lomsadze et al. 2005). The assembly completeness was calculated with BUSCO v3 (Simão et al. 2015), based on the alveolata_stramenophiles_ensembl data set. The BUSCO analysis produced the following percentage of complete or partially complete gene groups: CPV-219 = 91.5\%, CPV-262 $=72.6 \%$, CPV-267 $=82.9 \%$, CPV $-270=89.8 \%$, CPV-277 $=91.0 \%$, and CPV-302 $=93.2 \%$.

A core of 11,248 orthologs from the seven genomes was obtained using OrthoMCL ( $\mathrm{Li}$ et al. 2003). Two phylogenetic trees were obtained using 6,555 single-copy orthologous genes from our six genomes and the reference genome using MRBAYES with the evolution model GTR + G (Huelsenbeck and Ronquist 2001) and PhyML (maximum likelihood) (Guindon et al. 2010). Both trees showed an identical topology and indications of different relationships across core genes based on posterior probability and bootstrap support values. The majority of core genes did not cluster isolates by host or collection location.

Protein predictions were annotated by comparing our sequences against the Pfam database 32.0 (El-Gebali et al. 2019). Among the predicted proteins, we found an average of 78 genes belonging to an NPP1 Phytophthora toxins family. The total number of predicted proteins by genome were in the range of 19,275 to 25,102 . We retrieved high-quality RXLR and CRN protein sequences from the GenBank to create a protein model for each effector family. Protein models were created with hmm-build tool from HMMER v3.2.1. The proteome of each sequenced strain was scanned with the protein effector models, using $\mathrm{hmm}$-search from HMMER v3.2.1. We found an average of 345.8 RXLR and 185.8 CRN proteins, of which 1,756 RXLR and $430 \mathrm{CRN}$ are not in the reference genome. The effector sequences were deposited in the Database of Virulence Factors in Fungal Pathogens from the University of Nebraska-Lincoln (Lu et al. 2012). These data may reveal conserved and host-specific RXLR and CRN effectors, for which one could determine specific functions and mechanisms of action during the development of the disease in plants.

Data availability. This whole-genome shotgun project has been deposited at DDBJ/ ENA/GenBank under the accessions numbers RQEI00000000 (CPV-302), RQEJ00000000 (CPV-277), RQEK00000000 (CPV-270), RQEL00000000 (CPV-267), RQEM00000000 (CPV-262), and RQEN00000000 (CPV-219). The versions described in this article are the first versions. The reads for all isolates were deposited at NCBI/SRA under the accession number PRJNA505815.

\section{Author-Recommended Internet Resources}

alveolata_stramenophiles_ensembl data set: https://busco.ezlab.org/

HMMER v3.2.1:http://hmmer.org/ 


\section{Literature Cited}

Candole, B. L., Conner, P. J., McGregor, C., Waters, V., and Ji, P. 2012. The disease reactions of heirloom bell pepper "California Wonder" to Phytophthora capsici. Agric. Sci. 3:417-424.

El-Gebali, S., Mistry, J., Bateman, A., Eddy, S. R., Luciani, A., Potter, S. C., Qureshi, M., Richardson, L. J., Salazar, G. A., Smart, A., Sonnhammer, E. L. L., Hirsh, L., Paladin, L., Piovesan, D., Tosatto, S. C. E., and Finn, R. D. 2019. The Pfam protein families database in 2019. Nucleic Acids Res. 47:D427-D432.

Guindon, S., Dufayard, J. F., Lefort, V., Anisimova, M., Hordijk, W., and Gascuel, O. 2010. New algorithms and methods to estimate maximum-likelihood phylogenies: Assessing the performance of PhyML 3.0. Syst. Biol. 59:307-321.

Gurevich, A., Saveliev, V., Vyahhi, N., and Tesler, G. 2013. QUAST: Quality assessment tool for genome assemblies. Bioinformatics 29:1072-1075.

Hu, J., Shrestha, S., Zhou, S., Liu, X., and Lamour, K. 2018. Dynamic extreme aneuploidy (DEA) in the vegetable pathogen Phytophthora capsici sheds light on instant evolution and intractability. bioRxiv. doi:10.1101/297788

Huelsenbeck, J. P., and Ronquist, F. 2001. MRBAYES: Bayesian inference of phylogenetic trees. Bioinformatics 17:754-755.

Kajitani, R., Toshimoto, K., Noguchi, H., Toyoda, A., Ogura, Y., Okuno, M., Yabana, M., Harada, M., Nagayasu, E., Maruyama, H., Kohara, Y., Fujiyama, A., Hayashi, T., and Itoh, T. 2014. Efficient de novo assembly of highly heterozygous genomes from whole-genome shotgun short reads. Genome Res. 24:1384-1395.

Kong, L., Qiu, X., Kang, J., Wang, Y., Chen, H., Huang, J., Qiu, M., Zhao, Y., Kong, G., Ma, Z., Wang, Y., Ye, W., Dong, S., Ma, W., and Wang, Y. 2017. A Phytophthora effector manipulates host histone acetylation and reprograms defense gene expression to promote infection. Curr. Biol. 27:981-991.

Lamour, K. H., Mudge, J., Gobena, D., Hurtado-Gonzales, O. P., Schmutz, J., Kuo, A., Miller, N. A., Rice, B. J., Raffaele, S., Cano, L. M., Bharti, A. K., Donahoo, R. S., Finley, S., Huitema, E., Hulvey, J., Platt, D., Salamov, A., Savidor, A., Sharma, R.,
Stam, R., Storey, D., Thines, M., Win, J., Haas, B. J., Dinwiddie, D. L., Jenkins, J., Knight, J. R., Affourtit, J. P., Han, C. S., Chertkov, O., Lindquist, E. A., Detter, C., Grigoriev, I. V., Kamoun, S., and Kingsmore, S. F. 2012. Genome sequencing and mapping reveal loss of heterozygosity as a mechanism for rapid adaptation in the vegetable pathogen Phytophthora capsici. Mol. Plant-Microbe Interact. 25:1350-1360.

Li, L., Stoeckert, C. J., Jr., and Roos, D. S. 2003. OrthoMCL: Identification of ortholog groups for eukaryotic genomes. Genome Res. 13:2178-2189.

Lomsadze, A., Ter-Hovhannisyan, V., Chernoff, Y. O., and Borodovsky, M. 2005. Gene identification in novel eukaryotic genomes by self-training algorithm. Nucleic Acids Res. 33:6494-6506.

Lu, T., Yao, B., and Zhang, C. 2012. DFVF: Database of fungal virulence factors. Database (Oxford) 2012:bas032.

Pryszcz, L. P., and Gabaldón, T. 2016. Redundans: An assembly pipeline for highly heterozygous genomes. Nucleic Acids Res. 44:e113.

Reis, A., Paz-Lima, M. L., Moita, A. W., Aguiar, F. M., Fonseca, M. E. N., Café-Filho, A. C., and Boiteux, L. S. 2018. A reappraisal of the natural and experimental host range of neotropical Phytophthora capsici isolates from Solanaceae, Cucurbitaceae, Rosaceae and Fabaceae. J. Plant Pathol. 100:215-223.

Saghai-Maroof, M. A., Soliman, K. M., Jorgensen, R. A., and Allard, R. W. 1984. Ribosomal DNA spacer-length polymorphisms in barley: Mendelian inheritance, chromosomal location, and population dynamics. Proc. Natl. Acad. Sci. U.S.A. 81:8014-8018

Simão, F. A., Waterhouse, R. M., loannidis, P., Kriventseva, E. V., and Zdobnov, E. M. 2015. BUSCO: Assessing genome assembly and annotation completeness with single-copy orthologs. Bioinformatics 31:3210-3212.

Stam, R., Jupe, J., Howden, A. J. M., Morris, J. A., Boevink, P. C., Hedley, P. E., and Huitema, E. 2013. Identification and characterisation CRN effectors in Phytophthora capsici shows modularity and functionality diversity. PLoS One 8: e59517. 